This item is the archived peer-reviewed author-version of:

\title{
Stabilization and encapsulation of gold nanostars mediated by dithiols
}

\section{Reference:}

Wang Yusong, Belén Serrano Ana, Sentosun Kadir, Bals Sara, Liz-Marzán Luis M..- Stabilization and encapsulation of gold nanostars mediated by dithiols

Small - ISSN 1613-6810 - 11:34(2015), p. 4314-4320

Full text (Publishers DOI): http://dx.doi.org/doi:10.1002/smll.201500703

To cite this reference: http://hdl.handle.net/10067/1275710151162165141 


\title{
Stabilization and Encapsulation of Gold Nanostars Mediated by Dithiols
}

\author{
Yusong Wang ${ }^{1}$, Ana Belén Serrano ${ }^{1}$, Kadir Sentosun ${ }^{2}$, Sara Bals ${ }^{2}$, and Luis M. Liz- \\ Marzán ${ }^{1,3}$ \\ 1Bionanoplasmonics Laboratory, CIC biomaGUNE, Paseo de Miramón 182, \\ 20009 Donostia - San Sebastián, Spain \\ ${ }^{2}$ EMAT-University of Antwerp Groenenborgerlaan 171, B-2020 Antwerp , \\ Belgium
}

${ }^{3}$ Ikerbasque Basque Foundation for Science 48013 Bilbao, Spain

\begin{abstract}
Surface chemistry plays a pivotal role in regulating the morphology of nanoparticles, maintaining colloidal stability, and mediating the interaction with target analytes toward practical applications such as surface-enhanced Raman scattering (SERS)-based sensing and imaging. The use of a binary ligand mixture composed of 1,4benzenedithiol (BDT) and hexadecyltrimethylammonium chloride (CTAC) to provide gold nanostars with long-term stability is reported. This is despite BDT being a bifunctional ligand, which usually leads to bridging and loss of colloidal stability. It is found however that neither BDT nor CTAC alone are able to provide sufficient colloidal and chemical stability. BDT-coated Au nanostars are additionally used as seeds to direct the encapsulation with a gold outer shell, leading to the formation of unusual nanostructures including semishell-coated gold nanostars, which are characterized by high-resolution electron microscopy and electron tomography. Finally, BDT is exploited as a probe to reveal the enhanced local electric fields in the different nanostructures, showing that the semishell configuration provides significantly high SERS signals as compared to other core-shell configurations obtained during seeded growth, including full shells.
\end{abstract}




\section{Introduction}

Among a variety of possible nanoparticle morphologies, colloidal gold nanostars (AuNS) comprising a central core from which multiple sharp branches protrude, have been pinpointed as highly efficient surface-enhanced Raman scattering (SERS) substrates due to their tunable localized surface plasmon resonances (LSPR) and nanoantenna effects by controlling core size, along with tip length and sharpness.[1-8] A number of methods have been reported for the synthesis of AuNS, through either seedless or seed-mediated approaches, using capping molecules such as poly(N-vinylpyrrolidone) (PVP) or cetyltrimethylammonium bromide $(\mathrm{CTAB})$, but also under surfactant-free conditions.[2,7,9-11] Whereas PVP-capped AuNS can be synthesized with tailored dimensions and optical properties,[2,12] and used for ultrasensitive SERS detection,[13] the SERS signal is affected by hindered analyte chemisorption due to the adsorbed polymer, which can be removed only via a time-consuming process.[3] As an alternative, surfactant-free AuNS are of high interest considering that their cleaner surfaces are more suitable for surface functionalization, additionally avoiding toxicity issues derived from the surfactant.[11,14] However, these AuNS typically display poor stability, resulting in changes of the particles' morphology (reshaping) and corresponding LSPR blueshifts, which has also been reported for other nanostructures with sharp tips. $[15,16]$ In the absence of capping molecules, colloidal stability is also compromised, as van der Waals attractions become dominant, eventually leading to particle aggregation.[17] These observations clearly indicate that the surface properties of AuNS play a critical role on the colloidal and chemical stability, which is crucial for SERS-relevant applications.[18]

Interestingly, various strategies have been developed to functionalize the AuNS surface, so as to mediate the interaction of the nanoparticles with target analytes toward practical SERS applications.[18] For instance, the introduction of capping 
molecules such as polymer stabilizers,[2] thiolated polyethylene glycol,[11] surfactants, $[19,20]$ and proteins such as bovine serum albumin,[20] as well as protective inorganic shells, $[21,22]$ was used to improve colloidal stability prior to eventual application. A conceptually different strategy concerns the use of reduced graphene oxide (rGO) as support, with no need for additional polymer stabilizer or surfactant during AuNS synthesis.[17] Alternatively, an additional metal layer directly coated on the AuNS surface has been recently employed to develop stable SERS probes.[19] Additional NS-templated silver overgrowth produces hybrid particles and enables stronger SERS signal upon adsorption of Raman tags on the outer surface. However, few attempts have been directed toward incorporating the Raman tag between metal layers within the same particle, so as to generate plasmonic hot spots for the development of highly efficient SERS probes.[23-26]

We herein propose the use of 1,4-benzenedithiol (BDT) together with hexadecyltrimethylammonium chloride (CTAC), with the aim of stabilizing AuNS by avoiding both reshaping and aggregation, as well as directing the seeded growth of a gold shell around BDT-primed AuNS seeds, which may incorporate intrinsic hot spots. Electron tomography, SERS, and UV-vis spectroscopy show that various exotic nanostructures were obtained, all of them containing embedded BDT Raman tags. Interestingly, we find that semishell-coated nanostars display significantly enhanced SERS, as compared to other coated nanostructures and to nanostars with no coating shell.

\section{Results and Discussion}

\subsection{Morphological and Colloidal Stability of Gold Nanostars}

Surfactant-free AuNS were prepared following a seeded- growth protocol reported by Vo-Dinh and co-workers using citrate-coated spheres as seeds (Figure S1, Supporting Information).[11] The stability of the as-synthesized colloidal system was studied by comparing the effect of different capping molecules on the optical and morphological features of the particles. Figure 1 shows the UV-vis spectra of AuNS 
$(\approx 23 \mathrm{~nm}$ core size) after incubation (30 $\mathrm{min})$ with different capping molecules, including CTAC, BDT, and a CTAC/BDT mixture. The first observation from this graph is that uncapped AuNS undergo rapid transformation as indicated by a strong blueshift of the LSPR band, which was confirmed by transmission electron microscopy (TEM) analysis showing that aged particles feature rounded tips (Figure 1b). A similar effect (though not so pronounced) was found for AuNS that were incubated with CTAC (LSPR maximum $\approx 660 \mathrm{~nm}$ ), whereas BDT leads to a broadened LSPR band centered around $830 \mathrm{~nm}$, indicating aggregation that was confirmed by visual observation (see photograph in the inset of Figure 1a). In contrast, upon incubation with a BDT/CTAC mixture, the width of the LSPR band was preserved, which is in agreement with TEM observation of sharp nanostar spikes (Figure 1b). Comparison with other common capping ligands such as thiolated polyethylene glycol (PEG-SH, O-[2-(3-mercapto-propionylamino)ethyl]-O'methylpolyethylene glycol), 4-ami-nothiophenol (ATP), 4-mercaptobenzoic acid (MBA), and bis(p-sulfonatophenyl) phenylphosphine dihydrate dipotassium salt (BSPP) (Figure S2, Supporting Information) resulted in significant blueshifts and reshaping for all of these ligands.

The evolution of the different samples was monitored by UV-vis spectroscopy (see Figure S3, Supporting Information), which confirms that, whereas the LSPR band of the colloid comprising bare gold nanostars rapidly blueshifts, along with a decrease in extinction intensity and a dramatic change after $24 \mathrm{~h}$, introduction of CTAC slows down the blueshift rate. When a mixture of BDT and CTAC was added, the blueshift of the LSPR band was immediately quenched and the LSPR peak intensity was maintained. Such a significant improvement of AuNS stability upon addition of a thiolated molecule is likely due to the formation of Au S bonds that limit the mobility of $\mathrm{Au}$ surface atoms, as recently reported for mercaptopropionic acid.[27] It is interesting to note however that, as compared with monothiolated molecules(e.g., ATP and MBA, see Figure S2, Supporting Information), the dithiolated BDT was found to provide the longest LSPR stability, possibly because of the higher probability of $\mathrm{Au}$-thiol interaction. We postulate that the role of CTAC is the effective stabilization of the colloidal system, since AuNS capped with CTAC only display a 
narrow LSPR band (no aggregation) but significantly blueshifted because of reshaping (Figure 1). This synergistic effect combing the attributes of both the thiolated ligand and the surfactant was observed not only for gold nanostars, but also for gold nanospheres,[23] arrowhead gold nanorods (polypeptide-SH and CTAB),[28] or standard nanorods (PEG-SH and CTAB).[29] The stability of BDTmodified AuNS in CTAC is in fact surprising, as in general dithiol ligands are likely to promote bridging of Au NPs into aggregates, but obviously the presence of CTAC screens the potential binding of BDT with AuNS, and ultimately endows significant colloidal stability to the system. We thus conclude that, the binary mixture comprising BDT and CTAC shows an excellent capability to stabilize the freshly prepared gold nanostars.

\subsection{Encapsulation via Seeded Growth}

BDT-modified Au nanostars were used as seeds to direct further encapsulation within gold shells, considering that the strong binding between thiol groups and reduced gold atoms should affect the shell growth mode. Indeed, we found that standard seeded growth did not occur but instead a series of exotic gold nanostructures were obtained with distinct LSPR features (Figure 2), by simply tuning the concentration ratio $\left(R=\left[\mathrm{Au}^{3+}\right] /\left[\mathrm{Au}{ }^{0}\right]\right)$. Chemical reduction by ascorbic acid (AA) of increasing amounts of $\mathrm{HAuCl}_{4}$ on the capped AuNS led to a gradual decrease of the intensity of the band corresponding to the LSPR tip mode $(\approx 785$ $\mathrm{nm}$ ), while a higher energy band near the core mode emerged and progressively redshifted and increased in intensity. More specifically, for $R$ values higher than 4.8, the tip band was completely damped, with a dominant band centered at 560 $\mathrm{nm}$. The corresponding TEM images in Figure $2 \mathrm{~b}$ indicate that for increasing $\mathrm{R}$ values the gold nanostars get gradually encapsulated within an external gold shell. For $\mathrm{R}$ $=4.8$ a small number of spikes are exposed outside of the shell and when the $R$ value increases up to 19.1, the gold nanostars seem to be fully covered by the shells (Figure 2b). The morphological evolution can thus explain the changes in the UV-vis spectra, which were also observed when using larger AuNS as seeds (core size $\approx 55$ $\mathrm{nm}$ ) (Figure S4, Supporting Information), both regarding morphological and optical 
changes. In this case however, at small $R$ values satellite nanostructures (big nanostar decorated with multiple gold spheres) were dominant, in contrast to the core-shell geometry obtained using small Nanostars as seeds. For large $R$ values however, similar core-shell configurations were obtained for larger nanostars.

As the observed morphologies were quite intriguing, high-angle annular dark field scanning transmission electron microscopy (HAADF-STEM) tomography analysis was additionally carried out to reveal the 3D morphology of the coated particles, so as to discern potential internal gaps and connecting bridges between the AuNS seed and the shell. It should be noted that the intensity of HAADF-STEM images scales with the square of the atomic number of the elements present in the particle and with the sample thickness. Hence, the internal gaps within the particle decrease the intensity level in these regions of the HAADF-STEM images and therefore can be clearly recognized. For consistency, six different nanoparticles were investigated using HAADF-STEM tomography. A representative visualization of a 3D reconstruction and a 2D slice through it are depicted in Figure 3.

The 3D visualization displays the internal gaps as bubble-like regions highlighted with an arrow (Figure 3a). The internal gaps can also be clearly observed in the $2 \mathrm{D}$ slice image, corresponding to dark regions between the seed and the shell (Figure $3 b)$, while the same image also shows the presence of linking bridges between the AuNS seed and the shell. An estimate of the internal gap distances from electron tomography images yielded an average value of $4.5 \mathrm{~nm}$, though obviously narrower gaps are also present at some areas. Incidentally, electron tomography confirmed that the seed was not located at the center of the encapsulated shell but rather at the edge, indicating that the shell growth started from one or more of the AuNS tips and then asymmetrically continued, gradually surrounding the seed. This model is consistent with the auto- catalytic nature of seeded growth as well as with the electron tomography images obtained for NS-seeded nanostructures at different growth stages (R values), which include dimer, semishell, and full shell morphologies (Figure 4). 
Further information regarding the growth pathway of the shell on AuNS is provided by the crystalline structure, which was investigated for selected particles by means of HAADF- STEM imaging at atomic resolution. High-resolution images and their corresponding fast Fourier transform (FFT) patterns, obtained from six different positions on a semishell- coated Au nanostar (Figure 5), revealed that the crystalline orientations are not identical, meaning that the particle was tilted to a different zone axis for each of the high-resolution images. This observation indicates that the growth of the shell may start from more than one spot on the original AuNS (likely several tips). It should however be noted that overlapping regions between parts of the seed and shell make it difficult to precisely determine where on the seed the shell growth started. Compared with previous work on core-shell particles with internal gaps mediated by ligands, such as DNA,[24,30,31] block copolymers[26] or

other small molecules,[23,25] we show for the first time this type of seeded growth at atomic resolution and in 3D, which helps us to better understand this unusual growth mode.

\subsection{Surface Enhanced Raman Scattering}

One of the attractive features of this intriguing morphology is the presence of internal gaps, which are likely to display enhanced electric fields, therefore acting as intrinsic hot spots for SERS.[6] Since BDT has a reasonably high Raman scattering cross section and it should be located precisely at the gaps between the AuNS core and the outer shell, it is suitable as an internal standard to test the efficiency of such intrinsic hot spots. SERS spectra from stable colloids $(785 \mathrm{~nm}$ excitation wavelength) were collected at constant BDT- modified AuNS concentration (based on particle number). Comparison of the $\mathrm{CH}$ bending mode (1072 $\left.\mathrm{cm}^{-1}\right)$ SERS intensity after background subtraction showed a gradual increase up to an $\mathrm{R}$ value of 9.6 , which yields a fourfold higher intensity as compared to the bare gold nanostar seeds $(R=0)$, even though the LSPR band of the seeds is in resonance with the laser excitation $(785 \mathrm{~nm}$ ) (Figure 6). A decrease in SERS intensity was however observed for $R$ values above 9.6. Interestingly, the maximum SERS intensity does not correspond to fully coated AuNS, even though we should 
have the largest number of available molecular nanogaps between the core and the shell. This may be due to damping of light transmission through the outer shell or to additional contributions to E-field enhancement in anisotropic morphologies such as partly coated AuNS. It thus appears that the morphology yielding the highest SERS intensity is the semishell-coated nanostar, which was also found for larger coated AuNS ( $\approx 55 \mathrm{~nm}$ core size) (Figure S5, Supporting Information). It is well known that nanostructures comprising sharp tips generate intense electric near field by concentrating light onto the tips ("nanoantenna effect"), which leads to a significant SERS enhancement.[6,32,33] In the case of semishell-coated AuNS, the semishell may direct the light toward the exposed core tips, further contributing to the overall near field enhancement.

Apart from intensity differences, the Raman shift of the $\mathrm{CH}$ bending mode peak $(\approx 1072 \mathrm{~cm}-1)$ shows differences of $\approx 4 \mathrm{~cm}-1$ for growing shells up to $R=4.8$, then shifting back to higher wavenumbers, as shown in Figure 6b. A similar behavior was observed for the phenyl ring stretching mode, where the peak at $\approx 1572 \mathrm{~cm}^{-1}$ for AuNS seeds $(\mathrm{R}=0)$ shifts to $\approx 1563 \mathrm{~cm}^{-1}$ when the SERS intensity is close to the maximum value, then shifting back to $1573 \mathrm{~cm}^{-1}$ for fully encapsulated AuNS $(R=19.1)$ (Figure S6a, Supporting Information). A similar trend was observed for the larger AuNS ( $\approx 55 \mathrm{~nm}$ core size) (Figure S6b, Supporting Information). The variation in Raman scattering frequency likely reflects the change of local environment around the BDT molecules interfacing different gold nanostructures. In a related report,[34] the orientation of 1,4-BDT relative to a gold surface was shown to affect the position of the phenyl ring stretching mode in the SERS spectrum, with lower wavenumbers for flat as compared to perpendicular orientation, due to different gold surface-ring m-orbital interactions. In our system, the growth of gold shells may generate a compression force that changes the BDT orientation from perpendicular to parallel relative to the gold seed surface, resulting in a shift of the Raman band (phenyl ring stretching mode) toward lower wavenumbers as the shell growth progresses. However, further growth of the gold shell would result in the uncovered BDT layer becoming more rigid and 
hard to bend or change the original orientation. Accordingly, BDT perpendicular orientation, even after full shell growth, plays a major effect on the Raman peak (phenyl ring stretching mode) and shifts back to higher wavenumbers. These preliminary data show that these particles comprising Raman tags interfacing gold nanostructures can probe the local E-field enhancement, as well as local environment changes.

\section{Conclusions}

In summary, we report a new strategy that combines an aromatic dithiol (BDT) and a surfactant (CTAC) to stabilize and encapsulate surfactant-free gold nanostars, even though the individual components cannot perform a comparable stabilizing effect. Seeded growth in the presence of BDT leads to a series of exotic nanostructures, in which internal gaps are consistently present, as confirmed by electron tomography. Optical characterization showed that semishell-coated nanostars display significant SERS intensity as compared to other geometries obtained along the seeded growth process. The roles of bifunctional ligand and surfactant in the gold nanostar-seeded growth are thus: (1) protecting the initial gold nanostars from reshaping and aggregation, (2) directing Au shell growth around the core, and (3) generating significantly enhanced SERS activity. Additionally, partially encapsulated nanostars display a Janus nature, with bare gold on one side and a thiol-modified surface on the other, which could be further modified with different functional groups/ additional Raman active molecules, thus showing promise as functional SERS-coded probes for various applications such as sensing or imaging.

\section{Experimental Section}

Materials: Hydrogen tetrachloroaurate (III) trihydrate (HAuCl4.3H2O, $\geq 99.9 \%$ ), silver nitrate $(\mathrm{AgNO}, \geq 99.0 \%)$, AA ( $\geq 99 \%)$, sodium citrate tribasic dihydrate $(\geq 98 \%)$, BDT, CTAC, O-[2-(3-mercaptopropionylamino)ethyl]-O'-methylpolyethylene glycol (PEG-SH, $5000 \mathrm{Mw}$ ), MBA, ATP, and BSPP were all purchased from Sigma-Aldrich. 
Hydrochloric acid solution (37 wt\%) was purchased from Panreac. Milli-Q water $(18.2 \mathrm{M} \Omega \mathrm{cm})$ was used in all experiments.

Synthesis of Gold Nanostars and Incubation with Different Capping Molecules: AuNS were prepared by a modified seed-mediated growth method.[11] Briefly, the seed solution was prepared by adding citrate solution ( $5 \mathrm{~mL}, 1 \mathrm{wt} \%)$ to boiling $\mathrm{HAuCl} 4$ solution $(95 \mathrm{~mL}, 0.5 \times 10-3 \mathrm{M})$ under vigorous stirring. After $15 \mathrm{~min}$ of boiling, the solution was cooled down to room temperature and then kept at $4{ }^{\circ} \mathrm{C}$ for long-term storage. The as-synthesized Au nanoparticle seeds had an average size of $14.8 \pm 1.5 \mathrm{~nm}$, with an LSPR maximum at $519 \mathrm{~nm}$ (Figure S1, Supporting Information). For Au NS synthesis, different amounts of the citrate-stabilized seed solution (final $[\mathrm{Au0}]=0.05 \times 10-3 \mathrm{M}$ for small AuNS with core/overall size ratio of 23 $\mathrm{nm} / 39 \mathrm{~nm}$, and $5 \times 10-6 \mathrm{M}$ for larger AuNS with core/ overall size ratio of $55 \mathrm{~nm} / 98$ $\mathrm{nm})$ were added to $\mathrm{HAuCl} 4(10 \mathrm{~mL}, 0.25 \times 10-3 \mathrm{M})$ solution containing $\mathrm{HCl}(10 \mu \mathrm{L}$, $1.0 \mathrm{M}$ ) in a glass vial at room temperature under moderate stirring. Quickly, AgNO3 $(100 \mu \mathrm{L}, 3 \times 10-3 \mathrm{M})$ and $\mathrm{AA}(50 \mu \mathrm{L}, 100 \times 10-3 \mathrm{M})$ were added simultaneously to the above solution. The solution rapidly turns from light red to blue or greenishblack, depending on the final concentration of Au0. Specifically, $1 \mathrm{~mL}$ and $100 \mu \mathrm{L}$ of gold nanoparticle seeds were added to the growth solution to obtain the small and the large AuNS, respectively. Immediately after synthesis, equal amounts of AuNS solution were transferred to different capping molecule solutions: BDT $(10 \times 10-6$ $M)$, CTAC $(1 \times 10-3 \mathrm{M})$, mixture of BDT $(10 \times 10-6 \mathrm{M})$ and CTAC $(1 \times 10-3 \mathrm{M})$, ATP $(10 \times 10-6 \mathrm{M}), \mathrm{MBA}(10 \times 10-6 \mathrm{M}), \mathrm{PEG}-\mathrm{SH}(10 \times 10-6 \mathrm{M})$, and BSPP $(10 \times$ 10-3 M), and incubated for $30 \mathrm{~min}$. Afterward, all sample solutions together with a control sample (AuNS without capping molecule incubation) were centrifuged twice, washed to remove the supernatant, and redispersed in CTAC solution $(5 \times 10-3 \mathrm{M}$ for BDT, CTAC, (BDT+ CTAC), MBA, and ATP) and water (PEG-SH, BSPP, and bare). Corresponding UV-vis spectra were obtained after washing. Kinetic studies were performed by monitoring the UV-vis spectra of freshly prepared AuNS ( $223 \mathrm{~nm}$ core size), incubated with different capping molecules for different times ranging from $\approx 1 \mathrm{~min}$ to $24 \mathrm{~h}$, immediately after synthesis and without washing. 
Gold Shell Growth Using AuNS-BDT as Seeds: The as-prepared AuNS modified by the BDT+CTAC binary mixture were used as seeds for gold shell growth. Typically, a growth solution containing CTAC $(310 \mu \mathrm{L}, 0.1 \mathrm{M}), \mathrm{HAuCl} 4$, and AA $(20 \mu \mathrm{L}, 1.0 \mathrm{M})$ was first prepared to obtain a transparent solution. Afterward, Au NS-BDT seed solution $([\mathrm{Au} 0]=0.48 \times 10-3 \mathrm{M})$ was added to the growth solution under vigorous mixing. The reaction solutions were left undisturbed overnight and then the product was washed twice by centrifugation and redispersion in CTAC solution ( $5 \times 10-3 \mathrm{M})$. The morphology of the nanostructures obtained by seeded growth was tuned by changing the $R$ value $(R=[A u 3+] /[A u 0])$ during the synthesis.

Instrumentation and Characterization: UV-vis spectroscopy (Agilent UV-Visible, ChemStation) was used to collect extinction spectra of colloidal solutions in $1 \mathrm{~mm}$ quartz cuvettes or $10 \mathrm{~mm}$ plastic cuvettes (kinetic studies). Conventional TEM images were obtained using a JEOL JEM-2010 operating at $120 \mathrm{kV}$. Tilt series for electron tomography were acquired using a FEI Tecnai G2 electron microscope operated at $200 \mathrm{kV}$, in combination with a Fischione 2020 single tilt holder. Projection images were collected over an angular range of $\pm 74^{\circ}$ with $3^{\circ}$ tilt increments. The tilt series were aligned using the FEI INSPECT 3D software and subsequently reconstructed via the simultaneously iterative reconstruction technique (SIRT) implemented in the ASTRA tomography toolbox.[35] High-resolution HAADFSTEM images were acquired using a double corrected Titan 50-80 microscope operated at $300 \mathrm{kV}$. A Raman- IR microscope (Renishaw in Via Raman spectrometer) was used for solution SERS studies under $785 \mathrm{~nm}$ laser excitation, using ethanol as the calibration sample. Glass vials (Thermo Scientific, National C4015-96, $1 \mathrm{~mL}$ clear sepcap vials, $8 \times 40 \mathrm{~mm}$ ) were used for all solution Raman measurements.

\section{Supporting Information}

Supporting Information is available from the Wiley Online Library or from the author

\section{Acknowledgements}


This work was funded by the European Commission (SAVVY). The authors acknowledge financial support from European Research Council (ERC Advanced Grant no. 267867- PLASMAQUO, ERC Starting Grant no. 335078-COLOURATOM). The authors also appreciate financial support from the European Union under the Seventh Framework Program (Integrated Infrastructure Initiative No. 262348 European Soft Matter Infrastructure, ESMI).

[1] A. Shiohara, Y. Wang, L. M. Liz-Marzán, J. Photochem. Photobiol. C 2014, $21,2$.

[2] P. S. Kumar, I. Pastoriza-Santos, B. Rodríguez-González, F. J. García de Abajo, L. M. Liz-Marzán, Nanotechnology 2008, 19, 015606.

[3] L. Rodríguez-Lorenzo, R. Alvarez-Puebla, F. J. García de Abajo, L. M. LizMarzán, J. Phys. Chem. C 2010, 114, 7336.

[4] C. Hrelescu, T. K. Sau, A. L. Rogach, F. Jäckel, J. Feldmann, Appl. Phys. Lett. 2009, 94, 153113.

[5] S. Mazzucco, O. Stéphan, C. Colliex, I. Pastoriza-Santos, L. M. LizMarzan, F. J. García de Abajo, M. Kociak, Eur. Phys. J.: Appl. Phys. 2011, $54,33512$.

[6] R. Alvarez-Puebla, L. M. Liz-Marzán, F. J. García de Abajo, J. Phys. Chem. Lett. 2010, 1, 2428.

[7] W. Lu, A. K. Singh, S. A. Khan, D. Senapati, H. Yu, P. C. Ray, J. Am. Chem. Soc. 2010, 132, 18103.

[8] F. Hao, C. L. Nehl, J. H. Hafner, P. Nordlander, Nano Lett. 2007, 7, 729.

[9] J. Xie, J. Y. Lee, D. I. C. Wang, Chem. Mater. 2007, 19, 2823.

[10] M. Schütz, D. Steinigeweg, M. Salehi, K. Kömpeb, S. Schlücker, Chem. Commun. 2011, 47, 4216. 
[11] H. Yuan, C. G. Khoury, H. Hwang, C. M. Wilson, G. A. Grant, T. Vo-Dinh, Nanotechnology 2012, 23, 075102.

[12] S. Barbosa, A. Agrawal, L. Rodríguez-Lorenzo, I. Pastoriza-Santos, R. A. Alvarez-Puebla, A. Kornowski, H. Weller, L. M. Liz-Marzán, Langmuir 2010, 26, 14943.

[13] L. Rodríguez-Lorenzo, R. A. Alvarez-Puebla, I. Pastoriza-Santos, S. Mazzucco, O. Stéphan, M. Kociak, L. M. Liz-Marzán, F. J. García de Abajo, J. Am. Chem. Soc. 2009, 131, 4616.

[14] T. Vo-Dinh, A. M. Fales, G. D. Griffin, C. G. Khoury, Y. Liu, H. Ngo, S. J. Norton, J. K. Register, H. N. Wang, H. Yuan, Nanoscale 2013, 5, 10127.

[15] L. Rodriguez-Lorenzo, J. M. Romo-Herrera, J. Perez-Juste, R. A. Alvarez-Puebla, L. M. Liz-Marzan, J. Mater. Chem. 2011, 21, 11544.

[16] J. Zeng, S. Roberts, Y. Xia, Chem. Eur. J. 2010, 16, 12559.

[17] Y. Wang, L. Polavarapu, L. M. Liz-Marzán, ACS Appl. Mater. Inter- faces 2014, 6, 21798.

[18] R. A. Alvarez-Puebla, L. M. Liz-Marzán, Chem. Soc. Rev. 2012, 41, [19] A. M. Fales, H. Yuan, T. Vo-Dinh, J. Phys. Chem. C 2014, 118, 3708

[20] H. Yuan, Y. Liu, A. M. Fales, Y. L. Li, J. Liu, T. Vo-Dinh, Anal. Chem.2012, 85, 208.

[21] L. Rodriguez-Lorenzo, Z. Krpetic, S. Barbosa, R. A. Alvarez-Puebla, L. M. Liz-Marzán, I. A. Prior, M. Brust, Integr. Biol. 2011, 3,922.

[22] A. M. Fales, H. Yuan, T. Vo-Dinh, Langmuir 2011, 27, 12186. [23] N. Gandra, S. Singamaneni, Adv. Mater. 2013, 25, 1022.

[24] D. K. Lim, K. S. Jeon, J. H. Hwang, H. Kim, S. Kwon, Y. D. Suh, J. M. Nam, Nat. Nanotechnol. 2011, 6, 452. 
[25] Y. Feng, J. He, H. Wang, Y. Y. Tay, H. Sun, L. Zhu, H. Chen, J. Am. Chem. Soc. 2012, 134, 2004.

[26] J. Song, B. Duan, C. Wang, J. Zhou, L. Pu, Z. Fang, P. Wang, T. T. Lim, H. Duan, J. Am. Chem. Soc. 2014, 136, 6838.

[27] M. Morales Vega, A. Bonifacio, V. Lughi, S. Marsi, S. Carrato, V. Sergo, J. Nanopart. Res. 2014, 16, 2729.

[28] Y. Wang, D. Aili, R. Selegård, Y. Tay, L. Baltzer, H. Zhang, B. Liedberg, J. Mater. Chem. 2012, 22, 20368.

[29] C. Hamon, S. Novikov, L. Scarabelli, L. Basabe-Desmonts, L. M. LizMarzán, ACS Nano 2014, 8, 10694.

[30] J. W. Oh, D. K. Lim, G. H. Kim, Y. D. Suh, J. M. Nam, J. Am. Chem. Soc. 2014, 136, 14052.

[31] B. Zhao, J. Shen, S. Chen, D. Wang, F. Li, S. Mathur, S. Song, C. Fan, Chem. Sci. 2014, 5, 4460.

[32] R. A. Alvarez-Puebla, E. R. Zubarev, N. A. Kotov, L. M. Liz-Marzán, Nano Today 2012, 7, 6 .

[33] R. A. Alvarez-Puebla, A. Agarwal, P. Manna, B. P. Khanal, P. Aldeanueva-Potel, E. Carbó-Argibay, N. Pazos-Pérez, L. Vigderman, E. R. Zubarev, N. A. Kotov, L. M. Liz-Marzán, Proc. Natl. Acad. Sci. US A 2011, $108,8157$.

[34] S. W. Joo, S. W. Han, K. Kim, J. Colloid Interface Sci. 2001, 240, 391.

[35] W. J. Palenstijn, K. J. Batenburg, J. Sijbers, J. Struct. Biol. 2011, 176, 250. 


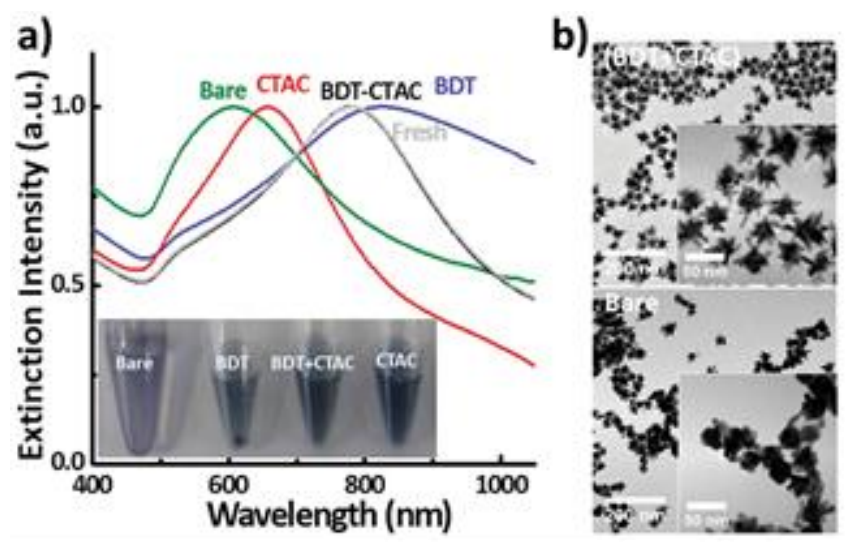

Figure 1. a) UV-vis spectra of $A u$ nanostars $(\approx 23 \mathrm{~nm}$ core size) after incubation with different capping molecules (CTAC, BDT, and BDT/ CTAC mixture) and control samples (Bare: without capping but similar processing; Fresh: spectrum recorded $\approx 40 \mathrm{~s}$ after the growth reaction without washing). Inset: Photographs taken $3 \mathrm{~h}$ after vortex shaking and gentle sonication of the corresponding colloids. b) TEM images of gold nanostars in the presence and in the absence of the BDT/CTAC mixture. 

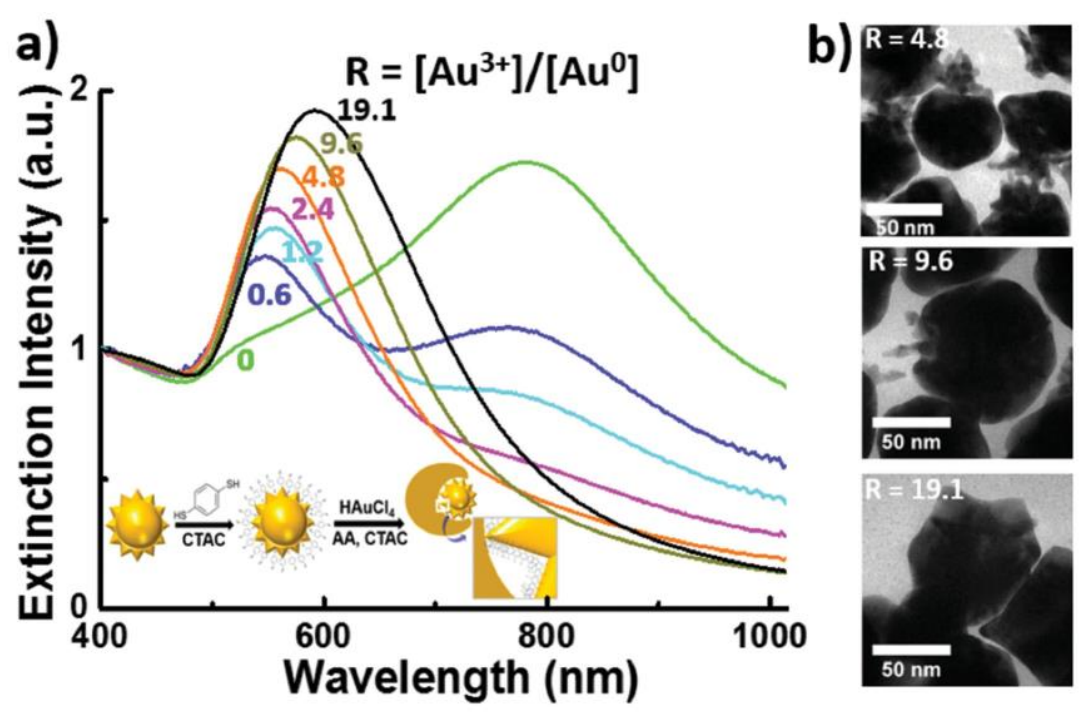

Figure 2. a) UV-vis extinction spectra of $A u$ nanostars ( $\approx 23 \mathrm{~nm}$ core size) during BDT- mediated seeded growth at different $R$ values $(R=[$ Au3 +$] /[$ Au0 $]=$ $0,0.6,1.2,2.4,4.8,9.6,19.1)$. The inset is a schematic illustration of the expected seeded growth, where CTAC and AA act as surfactant and reducing agent, respectively. b) TEM images of representative $A u$ nanostructures formed by reduction at different $R$ values. 

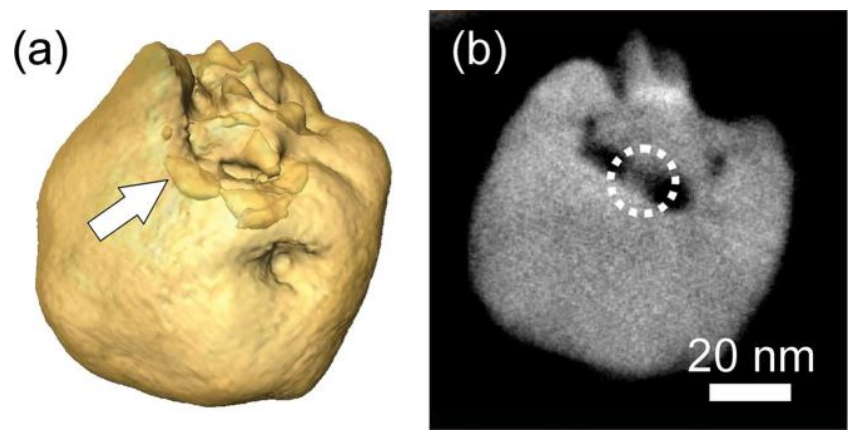

Figure 3. a) $3 D$ rendering of an electron tomography reconstruction for a semishell covered $\mathrm{Au}$ nanostar $(\approx 23 \mathrm{~nm}$ core size, $R=9.6)$. The gaps between the nanostar seed and the semishell can be clearly identified (example indicated by an arrow). b) A slice through the reconstruction reveals both the connections (highlighted with a dashed circle) and gaps between seed and shell. 


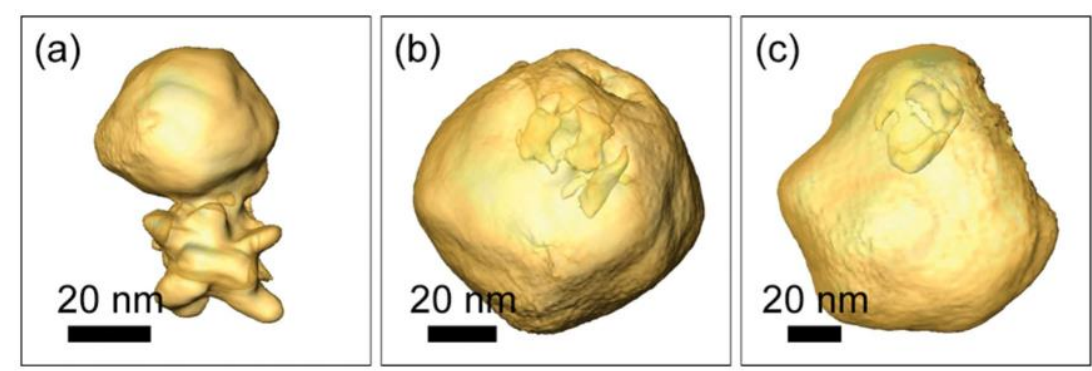

Figure 4. 3D rendering of electron tomography reconstructions of core-shell gold nanostars with increasing $R$ values: a) AuNS-sphere dimer $(R=4.8)$, b) semishell-coated AuNS $(R=9.6)$, c) encapsulated AuNS $(R=19.1)$. 


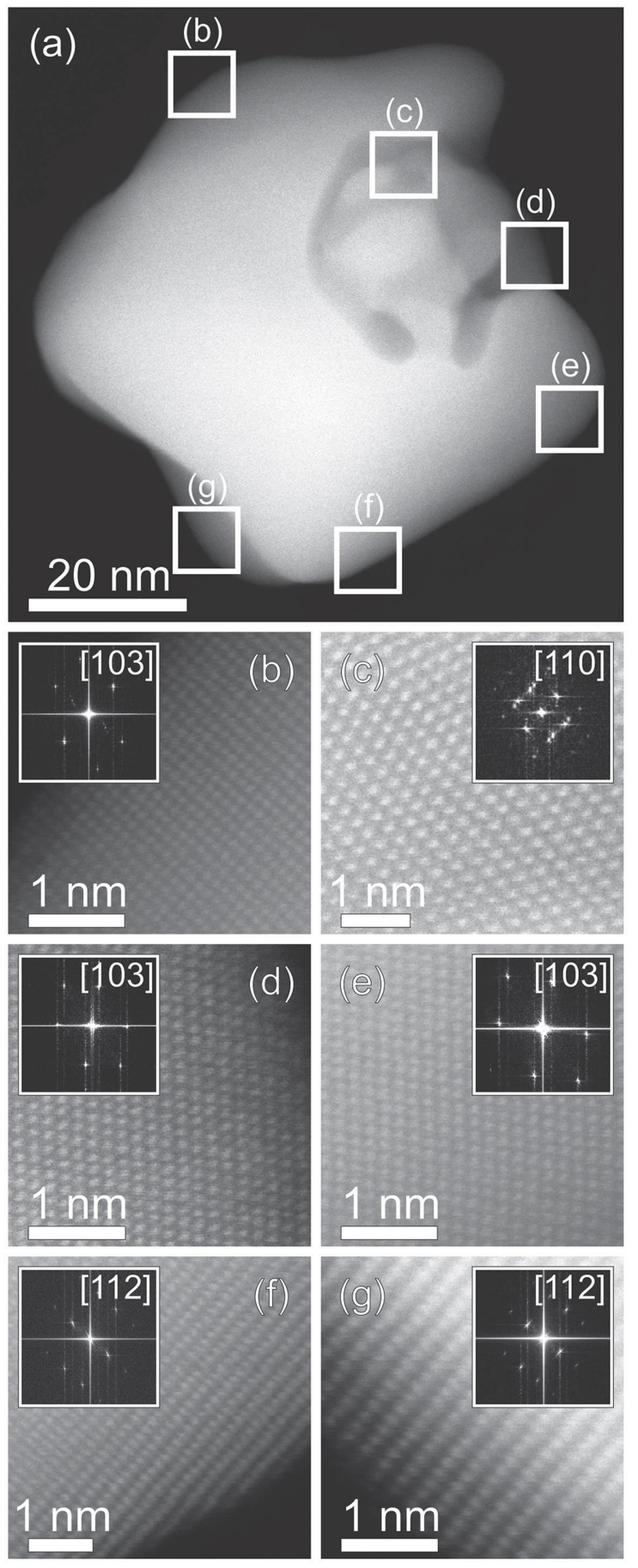

Figure 5. a) HAADF-STEM image providing an overview of a semishellcoated Au nanostar and b-g) high-resolution HAADF-STEM images collected from several regions highlighted in (a). The corresponding FFT patterns of the high-resolution HAADF-STEM images are displayed in the insets. 

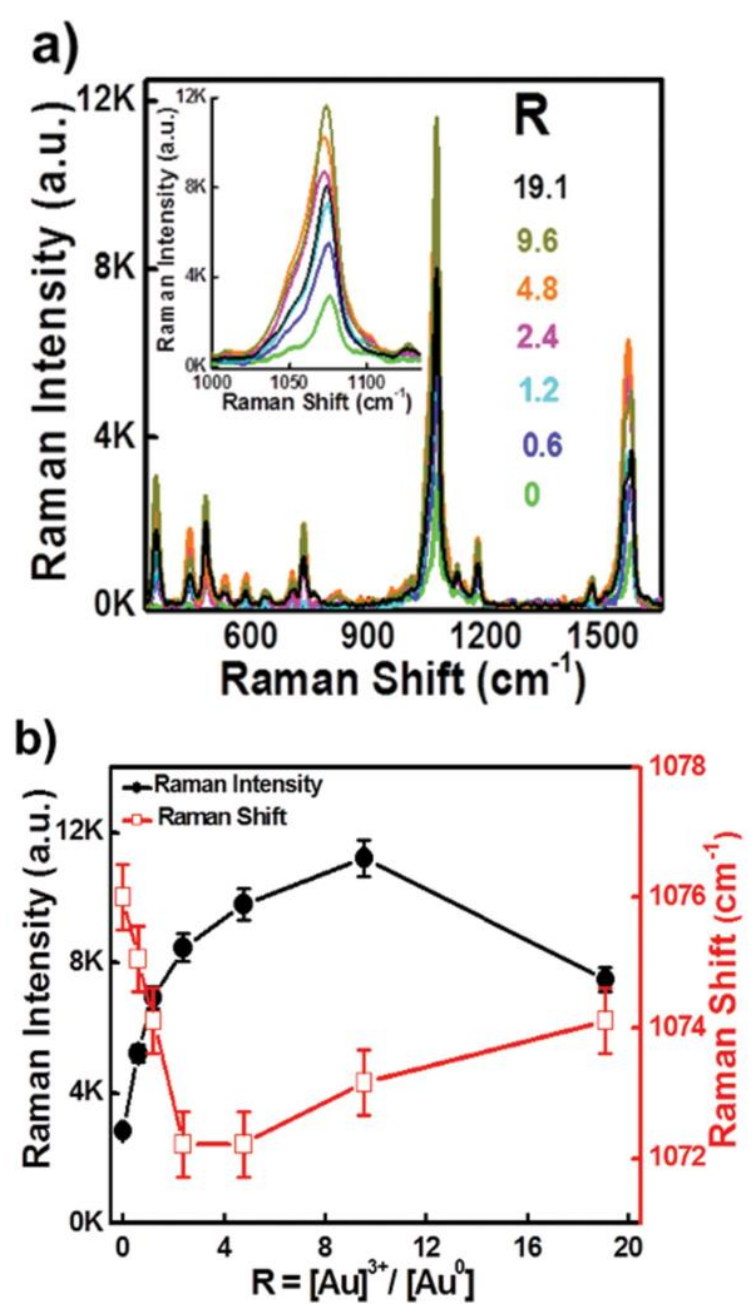

Figure 6. a) SERS spectra of BDT located within coated Au nanostars, synthesized at different $R$ values $(R=0.0,0.6,1.2,2.4,4.8,9.6,19.1)$. All samples have the same concentration of BDT-modified AuNS seeds. b) SERS intensity and position of BDT $\mathrm{CH}$ bending mode at different $\mathrm{R}$ values (background was subtracted). 